\title{
Efeito materno e paterno sobre as taxas de fertilização e eclosão em curimba (Prochilodus lineatus)
}

\author{
Maternal and paternal effect on fertilization and haching rate in \\ curimba (Prochilodus lineatus) \\ I.B. Allaman ${ }^{1}$, R.T.F. Freitas ${ }^{2}$, A.T.M. Viveiros ${ }^{2}$, A.F. Nascimento ${ }^{3}$, \\ G.R. Oliveira ${ }^{4}$, R.V. Reis Neto ${ }^{3}$ \\ ${ }^{1}$ Universidade Estadual de Santa Cruz - Ilhéus, BA \\ ${ }^{2}$ Universidade Federal de Lavras - Lavras, MG \\ ${ }^{3}$ Aluno de pós-graduação - Universidade Federal de Lavras - Lavras, MG \\ ${ }^{4}$ EPAMIG - Fazenda Experimental de Santa Rita - Prudente de Morais, MG
}

\begin{abstract}
RESUMO
Avaliou-se o quanto fêmeas e machos contribuem para a variação total das taxas de fertilização e de eclosão em curimba (Prochilodus lineatus). Utilizou-se sêmen criopreservado proveniente de cinco machos para fertilizar ovócitos de seis fêmeas em um esquema fatorial cruzado 5x6, totalizando 30 famílias. Além das características reprodutivas dos machos e fêmeas, foram avaliadas as taxas de fertilização e eclosão para cômputo dos efeitos materno e paterno. Os componentes da variância foram estimados por meio da máxima verossimilhança restrita, sendo construídos intervalos Highest Posterior Density (HPD) para cada componente. Verificou-se que as fêmeas contribuíram muito mais para a variação total em relação aos machos para as taxas de fertilização e eclosão. Para a taxa de fertilização, as fêmeas contribuíram com 26,3\% da variação total e os machos com 8,9\%. Em relação à taxa de eclosão, as fêmeas contribuíram com $11,9 \%$ e os machos com 1,6\%. Concluiu-se que houve efeito materno sobre as taxas de fertilização e eclosão e que o efeito paterno avaliado individualmente foi pouco expressivo ou até mesmo insignificante.
\end{abstract}

Palavras-chave: peixe, componentes da variância, efeito parental, índices reprodutivos

\begin{abstract}
The aim of this study was to evaluate how much females and males contribute to the total variation of reproductive traits such as fertilization and hatching rate in curimba Prochilodus lineatus. Cryopreserved semen from five males was used to fertilize eggs from six females in a cross-factorial 5x6, totaling 30 families. In addition to the reproductive characteristics of males and females, fertilization and hatching rates were evaluated for computation of maternal and paternal effects. The variance components were estimated by restricted maximum likelihood, and the Highest Posterior Density (HPD) intervals were estimated for each component. The female contributed more to the total variation than males for the fertilization and hatching rates. The female contributed $26.3 \%$ of the total variation in the fertilization rate against $8.9 \%$ of males. Regarding the hatching rate, the female contributed $11.9 \%$ versus $1.6 \%$ of males. Thus, there is maternal effect on rates of fertilization and hatching, and the paternal effect assessed individually was lackluster or even negligible.
\end{abstract}

Keywords: fish, variance components, parental effect, reproductive rates

Recebido em 9 de agosto de 2011

Aceito em 20 de julho de 2012

E-mail: ivanalaman@yahoo.com.br 


\section{INTRODUÇÃO}

O efeito materno pode ser definido como a contribuição, a influência ou o impacto sobre o fenótipo de um indivíduo, atribuível ao fenótipo da mãe, que podem ser causas genéticas ou ambientais (Hohenboken, 1984). Segundo Kamler (2005), o efeito materno contribui para a sobrevivência do embrião, que depende do estágio de maturação do ovo e da idade da fêmea via composição da matéria do ovo, resultando essas relações parental-ovo-progênie na variabilidade da larva em resistir a um período de fome e à predação.

As taxas de fertilidade e de eclosão são as primeiras características avaliadas nas fêmeas, sendo de suma importância em um processo de seleção de matrizes e reprodutores, pois sinalizam como está a qualidade do ovo, fator importante porque expressa a capacidade do ovo em produzir larvas viáveis (Kjorsvik et al., 1990).

A qualidade do ovo, especificamente a presença de vitaminas A, E, fatores imunes, antioxidantes e andrógenos (Garamszegi et al., 2007), pode ser afetada por diversos fatores, como idade da mãe, quantidade e qualidade da dieta (Lamontagne e McCauley, 2001; Dzyuba et al., 2006; Kyneb e Toft, 2006). Fêmeas menores produzem menor quantidade de ova e ovócitos de menor diâmetro (Johnston e Leggett, 2002), prejudicando, desse modo, a qualidade do ovo e, consequentemente, o desempenho e a sobrevivência das larvas e póslarvas (Wainhouse et al., 2001; Laugen et al., 2002; Higashitani et al., 2007).

Além da qualidade do ovo, o efeito paterno, representado pela qualidade do sêmen, também pode influenciar a taxa de fertilização e de eclosão (Weigensberg et al., 1998). A qualidade pode ser definida como a habilidade em fertilizar ovos (Aas et al., 1991) e é, provavelmente, intrínseca ou particular de cada indivíduo. Segundo Billard et al. (2004), a determinação da qualidade do sêmen é o passo fundamental para aperfeiçoar a fertilização, por meio da manipulação do sêmen, da seleção de reprodutores viáveis e de pesquisas com biotecnologias, tais como armazenamento de sêmen. No entanto, estudos que envolvam um delineamento complexo para detecção de efeitos parentais sobre indicadores reprodutivos são raros ou mesmo inexistentes.

Sendo assim, este estudo foi realizado com o objetivo de avaliar os efeitos materno e paterno sobre as taxas de fertilização e de eclosão em curimba Prochilodus lineatus.

\section{MATERIAL E MÉTODOS}

O experimento foi realizado na Estação de Hidrobiologia e Piscicultura da empresa Furnas Centrais Elétricas S/A, no estado de Minas Gerais, no distrito de Furnas, município de São José da Barra, entre os meses de janeiro e fevereiro de 2007. O município localiza-se geograficamente a $20^{\circ} 43^{\prime}$ de latitude sul e $46^{\circ} 18^{\prime}$ de longitude oeste.

Foram utilizados cinco reprodutores de curimba, da Estação de Piscicultura da Companhia Energética de Minas Gerais (Cemig). O sêmen coletado em um tubo de ensaio graduado foi congelado em glicose com metilglicol, segundo o protocolo estabelecido por Viveiros et al. (2009), para posterior utilização na fertilização. É importante ressaltar que o congelamento do sêmen para esse tipo de pesquisa é fundamental para que haja sincronização das fêmeas no momento da fertilização, uma vez que é necessário que os espermatozoides dos machos já estejam pré-disponíveis, pois a viabilidade dos ovócitos uma hora após a ovulação apresenta viabilidade reduzida (Andrade e Yasui, 2003). As matrizes de curimba foram provenientes da Estação de Hidrobiologia e Piscicultura de Furnas. O produto da desova de cada fêmea foi coletado em um béquer graduado e pesado em balança com precisão de $0,01 \mathrm{~g}$.

Cinco amostras de ovócitos da desova de cada fêmea, pesando aproximadamente $0,5 \mathrm{~g}$, foram coletadas em um recipiente de plástico e pesadas em balança digital semianalítica com precisão de $0,001 \mathrm{~g}$ para que os ovócitos fossem fertilizados com sêmen de cada macho que estava armazenado em palhetas de 0,5mL (Fig. 1), após descongelamento em banho-maria a $60^{\circ} \mathrm{C}$ por oito segundos (Viveiros et al., 2008). Para ativação do sêmen, foi utilizada solução $\mathrm{NaCl}$ $50 \mathrm{mM}$, na proporção de 1:5 (sêmen diluído:ativador) (Viveiros et al., 2009). 


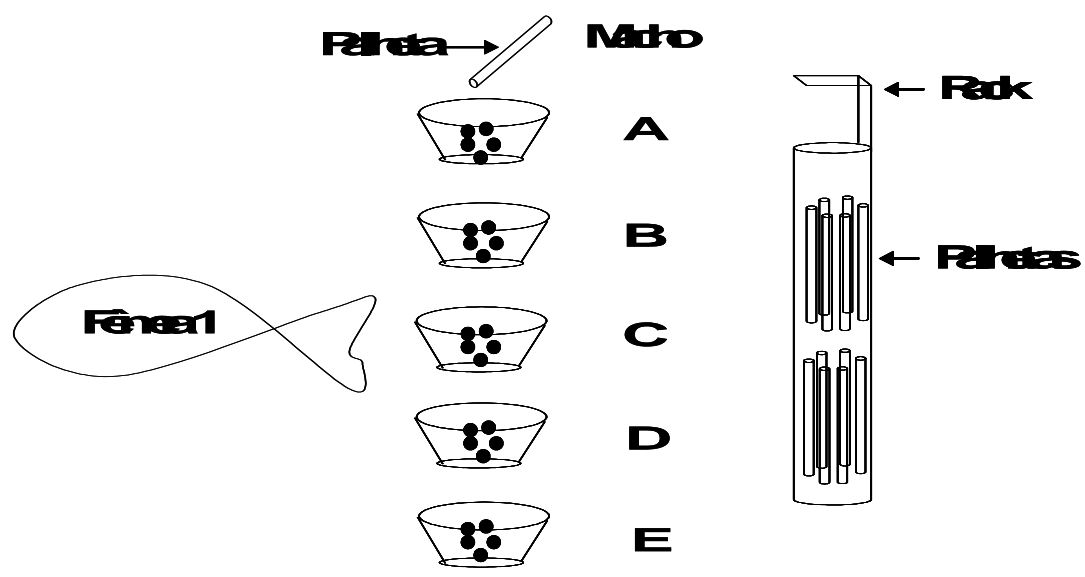

Figura 1. Esquema de fertilização em curimba (Prochilodus lineatus).

As 30 famílias geradas após a fertilização foram colocadas individualmente dentro de incubadoras experimentais de PVC (Viveiros et al., 2000), identificadas e distribuídas aleatoriamente dentro de um tanque de concreto azulejado, com troca de água contínua e constante. A taxa de fertilidade foi avaliada nove horas após a ativação dos gametas, em microscópio estereoscópico binocular, por meio da razão do número de embriões viáveis sobre a soma do número de embriões viáveis e inviáveis. A taxa de eclosão foi avaliada 22 horas após a ativação dos gametas (Ninhaus-Silveira et al., 2006), com temperatura média de $24^{\circ} \mathrm{C}$, por meio da razão do número de larvas eclodidas sobre a soma do número de larvas eclodidas e não eclodidas.

No decorrer do período experimental, foram mensurados os seguintes parâmetros da água: temperatura $\left(24,82 \pm 0,1^{\circ} \mathrm{C}\right), \quad \mathrm{pH} \quad(6,95 \pm 0,05)$, oxigênio dissolvido $\left(5,87 \pm 0,09 \mathrm{mg} \quad \mathrm{L}^{-1}\right) \quad$ e condutividade elétrica $\left(27,47 \mu \mathrm{S} \mathrm{cm}^{-1}\right)$.

As características avaliadas nas fêmeas foram: peso vivo - pesadas antes da indução hormonal, em uma balança digital regulada para quilogramas; peso da desova (PD) - coletada e pesada em balança digital regulada para gramas, no momento da desova; número de ovócitos por grama de ova (NO) - avaliados no momento da desova por meio da contagem de ovócitos de aparência branca e opaca (não viáveis) e ovos esverdeados (viáveis) em amostras correspondentes a um grama da desova total, para serem visualizadas em estereomicroscópio; e diâmetro dos ovócitos (DO) - após a pesagem e contagem dos ovócitos, avaliou-se uma amostra de 10 ovócitos por fêmea, aferida com auxílio de uma ocular micrométrica em estereomicroscópio do qual se obteve a média expressa em milímetros.

Nos machos, foram avaliados: peso vivo pesados em balança digital, antes que se fizesse a indução com hormônio, expresso em gramas; concentração espermática - estimada com uma câmara hematimétrica tipo de Neubauer Improved; e motilidade espermática - avaliada por meio da observação subjetiva de espermatozoides em movimento em relação ao total observado em microscópio com aumento de $400 \mathrm{X}$ e expressa em porcentagem.

O delineamento utilizado foi o inteiramente ao acaso, em esquema fatorial $6 \times 5$, sendo seis fêmeas e cinco machos, totalizando 30 famílias.

Considerando-se os efeitos de pai e mãe como aleatórios, os componentes da variância foram estimados por meio do método de máxima verossimilhança restrita, utilizando-se a função "lmer" do pacote "Ime4" (Bates et al., 2011). Foram construídos intervalos Highest Posterior Density (HPD) para os componentes da variância de cada fonte de variação, com o auxílio do método de Monte Carlo via Cadeias de Markov (MCMC), a fim de se alcançarem estimativas mais precisas. Utilizaram-se 10000 simulações para se obterem as estimativas. Os intervalos HPD foram obtidos por meio da função pvals.fnc do pacote languageR (Baayen, 2011). 
Utilizou-se $o$ software $\mathrm{R}$ versão 2.13 ( $\mathrm{R}$ Development Core Team, 2011) para a análise dos dados. Para a comparação dos componentes da variância das fontes de variação, foi estimada a participação percentual da variância da mãe, do pai e do erro em relação à variância total.

\section{RESULTADOS E DISCUSSÃO}

As médias e os erros-padrão das médias (EPM) dos dados do peso corporal e das características reprodutivas avaliados nos machos e nas fêmeas estão apresentados na Tab. 1.

Tabela 1. Dados das características reprodutivas de machos e fêmeas de curimba Prochilodus lineatus utilizados na formação das famílias

\begin{tabular}{lclc}
\hline Características & Média (EPM) & Características & Média (EPM) \\
\hline \multicolumn{1}{c}{ Macho } & & & Fêmea \\
\hline Peso vivo (kg) & $0,843(0,06)$ & Peso vivo (kg) & $2,172(0,292)$ \\
Volume seminal (ml) & $1,62(0,41)$ & Peso da desova (kg) & $0,358(0,037)$ \\
Concentração espermática & $5,392(0,387)$ & Diâmetro dos ovócitos (mm) & $0,681(0,01)$ \\
$\left(\right.$ sptz x $\left.10^{9} \mathrm{ml}^{-1}\right)$ & & &
\end{tabular}

Motilidade espermática

$72(3,74) \quad$ Número de ovos por grama de desova $\quad 1200,833(22,72)$

EPM: Erro-padrão da média.

Os valores das características reprodutivas estão próximos dos encontrados por Viveiros et al. (2010) para uma quantidade de amostra pequena e também estão dentro da faixa preconizada por Viveiros e Godinho (2009). No entanto, os valores de concentração espermática apresentados neste trabalho estão bem abaixo dos encontrados por Viveiros e Godinho (2009) e Viveiros et al. (2010).
A contribuição relativa da fêmea para variação total da taxa de fertilidade foi aproximadamente três vezes maior quando comparada à contribuição do macho. A fêmea contribuiu com $26,3 \%$ para variação total, e o macho com $8,9 \%$. Quanto à taxa de eclosão, a contribuição da fêmea para a variação total foi ainda maior, cerca de nove vezes maior do que a contribuição do macho, sendo $11,9 \%$ para fêmea e $1,6 \%$ para o macho (Tab. 2).

Tabela 2. Contribuição relativa da influência materna, paterna e da interação da taxa de fertilidade e da taxa de eclosão em curimba (Prochilodus lineatus)

\begin{tabular}{llcccc}
\multirow{2}{*}{ Característica } & Fontes & Variância & \multicolumn{2}{c}{ Intervalo HPD } & \multirow{2}{*}{$\begin{array}{c}\text { Contribuição } \\
\text { relativa (\%) }\end{array}$} \\
\cline { 4 - 6 } Taxa de fertilização & Fêmea & 0,00093 & 0,00000 & 0,00277 & 26,27 \\
& Macho & 0,00032 & 0,00000 & 0,00200 & 8,95 \\
\multirow{2}{*}{ Taxa de eclosão } & Fêmea & 0,00452 & 0,00000 & 0,00416 & 64,79 \\
& Macho & 0,00059 & 0,00000 & 0,02736 & 11,88 \\
& Erro & 0,03291 & 0,01682 & 0,00876 & 1,55 \\
& & 0,00229 & 0,00112 & 0,05551 & 86,57 \\
\hline
\end{tabular}

O maior índice de contribuição da mãe em relação ao pai, para variação total das taxas de fertilidade e eclosão, deve-se ao fato de a qualidade do ovócito ser mais impactante quando comparada à qualidade do sêmen. Uma vez que a fertilização e a eclosão são comumente avaliadas "x" horas após a fecundação, é provável que o sucesso da fertilização e da eclosão dependa mais do ovócito, influenciado pela idade, pelo peso e pela condição da mãe (Fox et al., 2003; Dzyuba et al., 2006) e, consequentemente, dos fatores contidos no ovócito, como nutrientes (Garamszegi et al., 2007), DNA mitocondrial (Brown et al., 2006), entre outros, em vez do sêmen propriamente dito.

A qualidade do sêmen, mensurada na maioria das pesquisas como motilidade e concentração 
espermática, é essencial para que ocorra a fecundação e está mais do que comprovada na literatura (Aas et al., 1991; Billard et al., 2004; Nascimento et al., 2010; Orfão et al., 2010; Viveiros et al., 2010). No entanto, este trabalho sugere que os pais individualmente contribuem apenas com os seus genes nucleolares e que o efeito, a influência ou a contribuição paterna mensurada como algo extragene nucleolar são tão pequenos quanto insignificantes no sucesso da fertilização e da eclosão. Gile e Ferguson (1995), ao avaliarem os fatores que afetam o potencial dos machos em gametas fecundados com pool de sêmen da espécie Oncorhynchus mykiss, atribuíram em um dos resultados que o diferencial na sobrevivência da progênie foi devido aos efeitos maternos ao invés dos efeitos paternos. Segundo Patton et al. (2007), a fonte materna geralmente tem um grande efeito sobre a viabilidade do embrião quando comparada ao macho, e a influência materna na qualidade dos ovos parece superar qualquer efeito de alelos recessivos deletérios herdados paternalmente.

A qualidade e a quantidade da dieta, embora não avaliadas neste estudo, são outros fatores importantes que influenciam na viabilidade dos embriões e, consequentemente, na sobrevivência de larvas e pós-larvas. Kyneb e Toft (2006) avaliaram a qualidade da dieta de fêmeas sob o desempenho de progênies da espécie Tachyporus hypnorum e concluíram que as progênies provenientes de fêmeas que receberam dietas de baixa qualidade tiveram um desempenho inferior quando comparadas às fêmeas que receberam dietas de alta qualidade. Um estudo com aranhas da espécie Dicymbium brevisetosum revelou que uma dieta materna de baixa qualidade nutricional reduziu a sobrevivência das progênies (Bilde e Toft, 2000).

A maior parte da variação total explicada pelo erro, provavelmente, deve-se ao fato de a interação fêmea $x$ macho estar confundida com o erro puro. Patton et al. (2007) encontraram uma interação significativa entre fêmea e macho em $50 \%$ das análises e comentaram que não se pode analisar viabilidade da prole observando simplesmente os efeitos de macho e fêmea individualmente. Saillant et al. (2001) estudaram a influência parental no desenvolvimento inicial da espécie Dicentrarchus labrax e encontraram interação significativa $(\mathrm{P}<0,01)$ entre fêmea e macho em todas as características avaliadas (taxa de fertilidade, taxa de sobrevivência, taxa de eclosão e comprimento total oito dias após eclosão). Acredita-se, também, que grande parte do erro se deva a fontes de variação que não foram mensuradas nesse estudo, como a capacidade de transmissão de antioxidantes, andrógenos, fatores imunes e de DNA mitocondrial (Hohenboken, 1984; Hiendleder e Wolf, 2003; Royle et al., 2003; Brown et al., 2006; Biard et al., 2007; Garamszegi et al., 2007; Gilbert et al., 2007).

\section{CONCLUSÃO}

Houve efeito materno sobre as taxas de fertilização e eclosão em curimbas, e o efeito paterno avaliado individualmente foi pouco expressivo ou até mesmo insignificante.

\section{AGRADECIMENTOS}

Os autores agradecem ao Conselho Nacional de Desenvolvimento Científico e Tecnológico (CNPq) pelo financiamento da bolsa de mestrado do primeiro autor.

\section{REFERÊNCIAS}

AAS, G.H.; REFSTLE, T.; GJERDE, B. Evaluation of milt quality of Atlantic salmon. Aquaculture, v.95, p.125-132, 1991.

ANDRADE, D.R.; YASUI, G.S. O manejo da reprodução natural e artificial e sua importância na produção de peixes no Brasil. Rev. Bras. Reprod. Anim., v.27, p.166-172, 2003.

BAAYEN, R.H. languageR: Data sets and functions with "Analyzing Linguistic Data: A practical introduction to statistics". 2011. Disponível em: <http://CRAN.R-project.org/package=languageR $>$. Acessado em: 10 mar. 2011.

BATES, D.; MAECHLER, M.; BOLKER, B. lme4: Linear mixed-effects models using S4 classes. 2011. Disponível em: <http://CRAN.Rproject.org/package=lme4>. Acessado em: 10 mar. 2011.

BIARD, C.; SURAI, P.F.; MOLLER, A.P. An analysis of pre- and pos-hatching maternal effects mediated by carotenoids in the blue tits. J. Evol. Biol., v.20, p.326339, 2007.

BILLARD, R.J.; COSSON, S.B.; NOVEIRI, M. Cryopreservation and short-term storage of sturgeon sperm, a review. Aquaculture, v.236, p.1-9, 2004. 
BILDE, T.; TOFT, S. Evaluation of prey for the spider Dicymbium brevisetosum Locket (Araneae: Linyphiidae) in single-species and mixed-species diets. Ekológia, v.19, p.9-18, 2000.

BROWN, K.H.; LEE, R.W.; THORGAARD, G.H. Use of androgenesis for estimating maternal and mitochondrial genome effects on development and oxygen consumption in rainbow trout, Oncorhynchus mykiss. Comp. Biochem. Physiol. Part B., v.143, p.415-421, 2006

DZYUBA, B.; LOOK, K.J.W.V.; CLIFFE, A. et al. Effect of parental age and associated size on fecundity, growth and survival in the yellow seahorse Hippocampus kuda. J. Exp. Biol., v.209, p.3055-3061, 2006

FOX, C.W.; BUSH, M.L.; WALLIN, W.G. Maternal age affects offspring lifespan of the seed beetle, Callosobruchus maculatus. Func. Ecol., v.17, p.811820, 2003.

GARAMSZEGI, L.Z.; BIARD, C.; EENS, M. et al. Maternal effects and the evolution of brain size in birds: Overlooked developmental constraints. Neurosci. Biobehav. Rev., v.31, p.498-515, 2007.

GILE, S.R.; FERGUNSON, M.M. Factors affecting male potency in pooled gamete crosses of rainbow trout, Oncorhynchus mykiss. Environ. Biol. Fish., v.42, p.267-275, 1995 .

GILBERT, L.; BULMER, E.; ARNOLD, K.E. et al. Yolk androgens and embryo sex: Maternal effects or confounding factors? Horm. Behav., v.51, p.231-238, 2007.

HIENDLEDER, S.; WOLF, E. The mitochondrial genome in embryo technologies. Reprod. Dom. Anim., v.38, p.290-304, 2003.

HIGASHITANI, T.; TAKATSU, T.; NAKAYA, M. $e t$ al. Maternal effects and larval survival of marbled sole Pseudopleuronectes yokohamae. J. Sea Res., v.58, p.78-89, 2007.

HOHENBOKEN, W.D. Maternal effects. In: CHAPMAN, A.B. General and quantitative genetics. Wisconsin: University of Wisconsin, 1984. cap.7, v.A4, p.135-147.

JOHNSTON, T.A.; LEGGETT, W.C. Maternal and environmental gradients in the egg size of an iteroparous fish. Ecology, v.83, p.1777-1791, 2002.

KAMLER, E. Parent-egg-progeny relationships in teleost fishes: an energetics perspective. Rev. Fish. Biol. Fish., v.15, p.399-421, 2005.

KJORSVIK, E.; MANGOR-JENSEN, A.Ç; HOLMEFJORD, I. Egg quality in fishes. Adv. Mar. Biol., v.26, p.71-113, 1990.
KYNEB, A.; TOFT, S. Effects of maternal diet quality on offspring performance in the rove beetle Tachyporus hypnorum. Ecol. Entomol., v.31, p.322330, 2006

LAMONTAGNE, J.M.; McCAULEY, E. Maternal effects in daphnia: what mothers are telling their offspring and do they listen? Ecol. Lett., v.4, p.64-71, 2001.

LAUGEN, A.T.; LAURILA, A.; MERILA, J. Maternal and genetic contributions to geographical variation in Rana temporaria larval life-history traits. Biol. J. Lin. Soc., v.76, p.61-70, 2002.

NASCIMENTO, A.F.; MARIA, A.N.; PESSOA, N.O. et al. Out-of-season sperm cryopreserved in different media of the Amazonian freshwater fish pirapitinga (Piaractus brachypomus). Anim. Reprod. Sci., v.118, p.324-329, 2010.

NINHAUS-SILVEIRA, A.; FORESTI, F.; AZEVEDO, A. Structural and ultrastructural analysis of embryonic development of Prochilodus lineatus (Valenciennes, 1836) (Characiforme; Prochilodontidae). Zygote, v.14, p.217-229, 2006.

ORFÃO, L.H.; MARIA, A.N.; NASCIMENTO, A.F. et al. Sperm fertility of the subtropical freshwater streaked prochilod Prochilodus lineatus (Characiformes) improved after dilution and cold storage. Aqua. Res., v.41, p.679-687, 2010.

PATTON, S.J.; KANE, S.L.; WHEELER, P.A. et al. Maternal and paternal influence on early embryonic survival of androgenetic rainbow trout (Oncorhynchus mykiss): Implications for measuring egg quality. Aquaculture, v.263, p.26-34, 2007.

R DEVELOPMENT CORE TEAM. $R$ : a language and environment for statistical computing. Vienna: $\mathrm{R}$ Foundation for Statistical Computing, 2009. Disponível em: <http://www.R-project.org>. Acessado em: 10 nov. 2009.

ROYLE, N.J.; SURAI, P.F.; HARTLEY, I.F. The effect of variation in dietary intake on maternal deposition of antioxidants in zebra finch eggs. Func. Ecol., v.17, p.472-481, 2003.

SAILLANT, E.; CHATAIN, B.; FOSTIER, A. et al. Parental influence on early development in the European sea bass. J. Fish Biol., v.58, p.1585-1600, 2001.

VIVEIROS, A.T.M.; GODINHO, H.P. Sperm quality and cryopreservation of Brazilian freshwater fish species: a review. Fish. Phys. Bioch.,v.35, p.137-150, 2009. 
VIVEIROS, A.T.M.; NASCIMENTO, A.F.; ORFÃO, L.H. et al. Motility and fertility of the subtropical freshwater fish streaked prochilod (Prochilodus lineatus) sperm cryopreserved in powdered coconut water. Theriogenology, v.74, p.551-556, 2010.

VIVEIROS, A.T.M.; ORFÃO, L.H.; MARIA, A.N. et al. A simple, inexpensive and successful freezing method for curimba Prochilodus lineatus (Characiformes) semen. Anim. Reprod. Sci., v.112, p.293-300, 2009.

VIVEIROS, A.T.M.; SO, N.; KOMEN, J. Sperm cryopreservation of African catfish (Clarias gariepinus): cryoprotectants, freezing rates and sperm: egg dilution ratio. Theriogenology, v.54, p.1305-1308, 2000 .
WAINHOUSE, D.; ASHBURNER, R.; BOSWELL, R. Reproductive development and maternal effects in the pine weevil Hylobius abietis. Ecol. Entomol., v.26, p.655-661, 2001.

WEIGENSBERG, I.; CARRIÉRE, Y.; ROFF, D.A. Effects of male genetic contribution and paternal investment to egg and hatchling size in the cricket, Gryllus firmus. J. Evolut. Biol., v.11, p.135-146, 1998. 